\title{
A method to reduce partial discharges in motor windings fed by PWM inverter
}

https://doi.org/10.1515/phys-2018-0078

Received November 2, 2017; accepted November 12, 2017

\begin{abstract}
A new approach for designing ac motor windings free of partial discharges (PD) is proposed. The method consists of adding a thin conducting layer on the outer surface of the enameled wire used for building the machine winding. With this additional layer, PDs occur only in critical zones localized near the wire connections rather than randomly in the residual voids between turns. With such deterministic localizations, it is possible to reduce strongly the PD activity by adding small quantities of varnish in the determined critical zones. After studying the validity of the Paschen's hypotheses for this application, the Paschen's law is coupled to an electrostatic finite element analysis, for predicting the Partial Discharge Inception Voltage (PDIV), which corresponds to the electronic avalanche ignition in the air of critical zones.
\end{abstract}

Keywords: AC machine windings, motor design, electric field, shielding layer, partial discharges, finite element simulation.

PACS: 84.30.Jc

\section{Introduction}

In recent airliners, the electrical power is increasing because this energy brings more flexibility and many other advantages comparing to hydraulic and pneumatic ones. Conventional aircraft have several grids. For increasing the electric power, the aircraft industry has chosen a higher voltage, switching from $115 \mathrm{~V}$ AC to $540 \mathrm{~V}$ DC. This solution yields a higher flexibility and reduces losses in cables. With this choice, high power density electric actuators are connected to the 540V DC grid by inverters that provide efficient machine controls, with a full reversibility of the

\footnotetext{
*Corresponding Author: Daniel Roger: Univ. Artois - EA4025, LSEE, 62400 Bethune - FRANCE, E-mail: daniel.roger@univ-artois.fr Sonia Ait-Amar, Ewa Napieralska: Univ. Artois - EA4025, LSEE, 62400 Bethune - FRANCE
}

power. However, the electronic power components of the inverters impose steep-fronted voltage pulses that cause repetitive short voltage spikes in the machine windings. These voltage spikes may cause PDs and an earlier aging of the machine. The aging mechanism is known for a long time [1, 2]. In the polymer based electrical insulation system (EIS), PDs in small air-voids of windings slowly erode the insulation layer. This phenomenon is due to voltage spikes exceeding the partial discharge inception voltage (PDIV) of the motor EIS [3, 4].

For permanent magnet synchronous motor (PMSM), the machine winding can be made with concentrated windings (one coil per stator slot). This topology makes it possible to design deterministic coils where the exact position of each turn is known. Therefore, a specific coil design may guarantee a good distribution of voltage spikes able to avoid PD [5].

The PMSM are excellent machines that can be used for many actuators, but they are expensive. The aircraft industry needs also cheaper machines for many actuators, in the air conditioning system for instance. Induction machines can perform these functions in very good economic conditions, but they need distributed windings. Their coils are made by automatic machines and inserted in the stator slots. The windings have a random topology. The turnto-turn voltage may be very large because the probability to have the input turn near the output one is not negligible [6]. For these parts of the coil, the thin insulating layer of the enameled wire and the residual air between them must withstand a high voltage stresses for inverter-fed motors. Therefore, it is impossible to guarantee the absence of PD in such random coils with a standard enameled wire. A classical solution consists in weakening the electric field between turns by using a thicker insulation layer, with the drawback of lower current density in the slots and heavier motors. Another solution consist in adding a filter that reduces the voltage fronts steepness but the filter is an additional component that has a mass and a cost.

The paper proposes another technological solution that consists of adding a thin conducting layer over the outer surface of the enameled wire. this layer creates equipotential surfaces that moves the critical zone, where 
DPs appear, toward deterministic areas. A local solution able to perform an insulation reinforcement in the critical zones is possible. The advantages, drawback and the technology readiness level (TRL) of the proposed solution are also discussed.

\section{Principle of the proposed technological solution}

The proposed method consist in adding a microscopic conducting layer on the outer part of the enameled wire used for building low voltage machines. This technology exist for large machines, it is used locally for getting a better potential distributions in end-winding connections $[7,8]$. In low voltage motor coils, the outer surfaces of the wires are in contact together. Consequently, the additional conducting layer creates equipotential surfaces that reduce strongly electric field in the air between the turns. The electric field is concentrated in the polymer covering the copper wires, which can withstand much higher electrical fields than air. With the thin conducting additional layer, the critical zones, corresponding to high field magnitudes in air, are moved towards the ends of the additional conducting layer situated near the connections of the coil. In these critical zones, the insulation must be reinforced to avoid PD for the highest voltage spikes. Investigations are made on twisted pairs made following the testing procedure defined in the IEC 60172 standard [9], that produce repetitive PDIV measurements in a given environment (temperature, pressure, humidity). In figure 1, the upper picture shows such a standard twisted pair and the lower one, shows a similar twisted pair with an additional thin external layer made of a silver conductive paint.

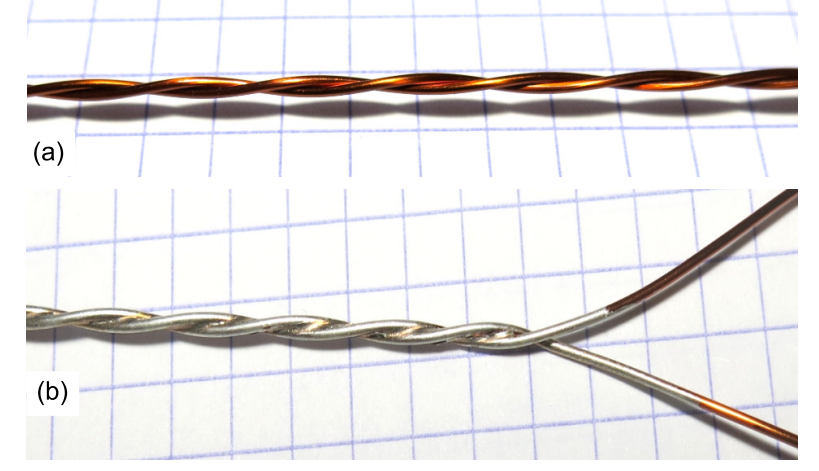

Figure 1: Standard twisted pair (a) and another one covered with a thin layer of silver conducting paint (b)
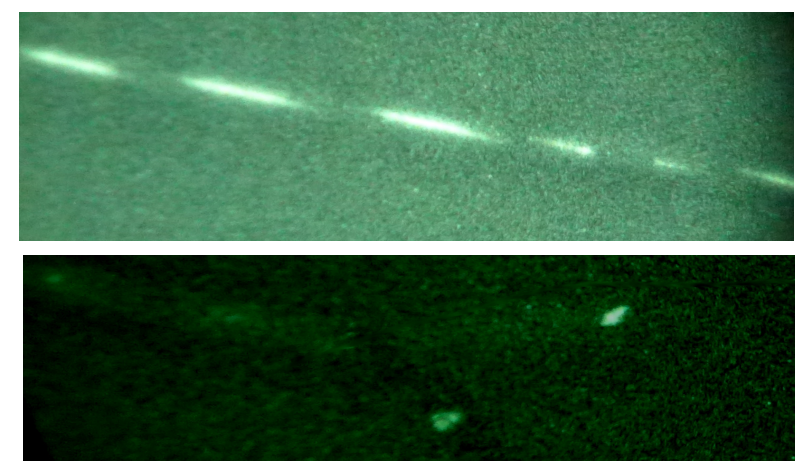

Figure 2: Pictures made on both twisted pairs in a deep darkness by a night vision device

For analyzing the influence of the thin additional layer of conducting paint on the electric field distribution, an experience has been carried out. Results are shown in figure 2 The pictures are obtained in a deep darkness, with a night vision device called "CoronaFinder" [10] placed at $0.8 \mathrm{~m}$ of the twisted pairs. This optical system is able to make a visible image from the UV radiation emitted by DPs. This night vision device is designed for being used at naked eyes; a standard camera is added to get the pictures with long exposure times. For this experience, a $50 \mathrm{~Hz}$ sine voltage of $1200 V_{\text {peak }}$ feeds the two twisted pair. This voltage is chosen over the PDIV for getting enough PDs. The PD level, measured electrically by a standard equipment, shows a similar PDs activities for both twisted pairs.

The upper photography of Fig. 2 corresponds to the standard twisted pair. The quality is low because of the general noise level. However, this picture shows that PDs are distributed along the twisted pair. The dark areas correspond to the shadows, made by the enameled wire situated between the PDs source, in the ambient air and the night vision device. At such a voltage, the electric field in the insulation layer is much under the polymer breakdown. The photography in the lower part of figure 2 is obtained in the same electrical condition, but with the twisted pair covered by a thin layer of silver conductive paint. On this picture, it is impossible to see the twisted pair body because of the darkness and the absence of PD in this area. Conversely, two bright points appear. They corresponds to many PDs at the ends of the thin layer of silver conductive paint.

Unfortunately, the night vision device associated to a standard camera cannot measure the energy of the PD radiation. However, the lower global noise of the picture suggests that the bright points correspond to a higher radiated energy in small zones. This photography suggests the ex- 


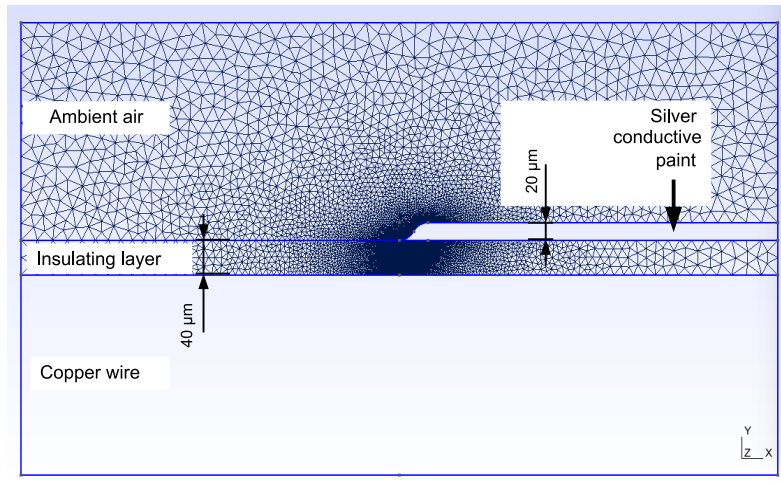

Figure 3: Geometry and mesh of the polymer layer and the ambient air in the critcial zone where PDs may appear

istence of a concentration of a similar PD activity, in much smaller areas than without conducting paint.

\section{Elecrtic field in the critical zones}

\subsection{Geometry of the critical zone}

A finite-element electrostatic simulation is performed for predicting the PDIV in the critical zones where PDs may appear. The conducting layer covering the enameled wire moves these critical zones toward the ends of the wire. The conducting regions are not meshed; they form the voltage source of the electrostatic problem. The electric fields are computed in the polymer layer and in the ambient air. Figure 3 shows the 2D finite element model of the electrostatic problem built at the micrometer scale. A dense mesh is applied in the critical zone of air, near the end of the additional conducting layer.

\subsection{Results and discussions}

Figure 4 presents the color map of the electric field in the critical zone of air for $500 \mathrm{~V}$ applied between the copper wire and the silver conducting paint. This voltage corresponds to half the peak voltage of the PDIV measured on twisted pairs. Indeed, when twisted pairs are supposed to be made of two identical enameled wires covered by an equipotential conducting layer, the voltage applied between the too copper conductors is split in two equal parts. The highest field values, where PDs may appear, are concentrated near the end of the silver conducting paint. A detailed analysis of the FE simulation results can be made using Paschen theory, for predicting the PDIV of the twisted

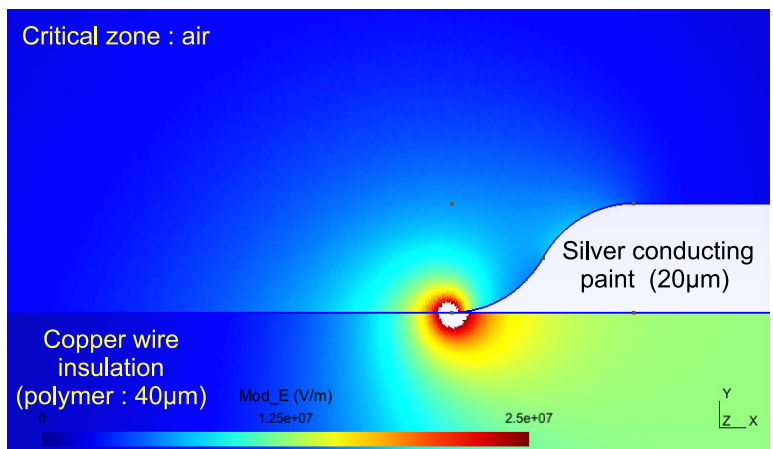

Figure 4: Geometry and mesh of the polymer layer and the ambient air in the critical zone where PDs may appear

pair made by an enameled wire covered by a conducting paint.

Let us remind that, more than a century ago, Paschen studied the ignition of the ionization of a pressurized gas, under an electric field [11]. At the same period, Townsend worked on gas ionization processes, where free electrons are accelerated by electric fields [12]. Similar studies were performed for typical non-uniform fields due to specific electrode shapes used for high-voltage applications [13]. These approaches are based on the acceleration of free electrons, in the mean free path between gas molecules at a given pressure. When the free path is too short (high pressures), the electron gives up its energy in a series of non-ionizing collisions. When the mean free path is too long (low pressures), the electron reaches the anode before colliding with a gas molecule. In both cases, there is no gas ionization. The electronic avalanche occurs when the accelerated free electrons have enough energy for ionizing gas molecules when they collide. Then, a complex chain reaction leads breakdown, if the electric field remains high enough [14].

The Paschen's empiric law defining the breakdown voltage in a gas is still a reference. This law was built on the results of many experiences made with two plane metallic electrodes in a pressurized gas. Therefore, the electric fields of Paschen's experiences are uniform; free electrons accelerate along straight lines toward the anode. In practical cases, the electric field is never uniform. Figure 5 shows the field lines in the critical zone of air for our problem. The field lines are curved; the field in not uniform.

The interesting part of each field line starts from the polymer surface and finishes on the silver conducting paint. Unlike Paschen's experiments, the electron inertia may have an influence: it is not obvious that the free electrons follow the field lines. The consequences of this major difference must be carefully studied before applying 


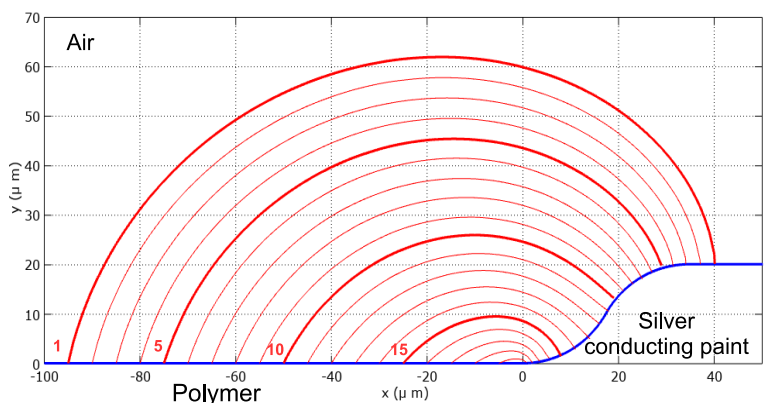

Figure 5: Field lines in the critical zone of air

Paschen's law. Two basic questions must be considered:

- Do free electrons follow a filed line as they do in the Paschen's experiences?

- If the first answer is "yes", a second question arises: does the variations of the field magnitude along a field line influence the Paschen's threshold?

For answering the first question, the ionization energy of molecules must be considered ( $13.6 \mathrm{eV}$ for oxygen and $14.5 \mathrm{eV}$ for nitrogen). It is supposed that the voltage is equal to the PDIV. For this voltage corresponding to the very beginning of the electronic avalanche. This hypothesis can be formulated in other words considering a microscopic point of view. It is supposed that the electric field accelerates a free electron until it acquires a kinetic energy equal to the gas molecule ionization one. Then a collision with a molecule absorbs this energy for creating a new free electron, which is also accelerated by the field. The random aspects of the problem are also neglected.

With this simplifying hypothesis, it is possible to estimate the trajectory of successive free electrons by solving the mechanical equation of a solid charged object placed in the field force $(\vec{F}=q \vec{E})$, defined in the critical zone (the electron mass is $m=9.10910^{-31} \mathrm{~kg}$ and its charge $\left.q=-1.60210^{-19} \mathrm{C}\right)$. The hypotheses suppose that the gas pressure is adapted to the voltage imposed on the twisted pair : the ionizing collisions occur exactly when free electrons have enough kinetic energy.

Figure 6 shows simulation results of the mechanical problem, that consider the mass of moving electrons. A free electron at zero speed is supposed to appear at the origin of the filed line 15 of figure 5 Two voltage levels are considered: $500 \mathrm{~V}$, which correspond to the PDIV in air at atmospheric pressure, and half of this value that corresponds to a lower pressure. The time step used for solving the mechanical equation is $0.01 \mathrm{ps}$ and the geometry of the critical zone is a square mesh of $0.5 \mu \mathrm{m}$.

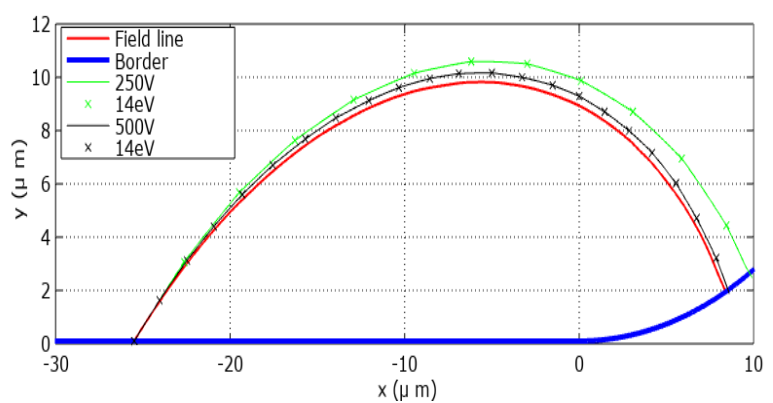

Figure 6: Trajectories of free electrons for two voltages and the field line 15 copied from Fig. 5

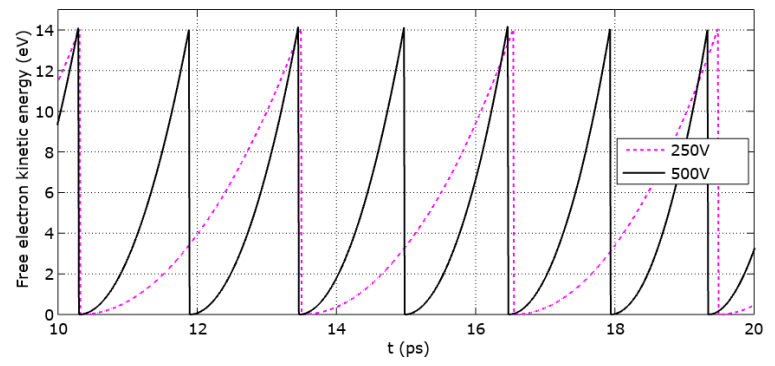

Figure 7: Kinetic energy of free electrons between collisions for two voltages and the field line 15

The small crosses plotted on figure 6 correspond to the ionizing collisions with a $\mathrm{N}_{2}$ or a $\mathrm{O}_{2}$ molecule considering an average energy of $14 \mathrm{eV}$. The electron trajectories cumulated from the starting point on the polymer layer toward the end point on the silver paint. The successive electron trajectories are not exactly superimposed to the field lines; the difference is higher for lower fields because the Coulomb forces are lower for the same inertial ones. For instance, at $250 \mathrm{~V}$ and for a cumulated electron trajectory that starts at the beginning of the field line 15 , the trajectory length is $41.5 \mu \mathrm{m}$ (1.5 $\mu \mathrm{m}$ longer than the field length). This cumulated trajectory is the same that the field line for $500 \mathrm{~V}$ (the difference is lower than the mesh grid). Figure 7 shows that, for lower fields, free electrons need more time, and consequently more space, for acquiring the ionization energy of $14 \mathrm{eV}$.

This simplified approach shows that, for the considered geometry, the field line curvatures can be neglected at ambient pressure, but not for very low pressures.

The energy acquired by an accelerated free electron is a key value for answering the second question relative to the influence of the variations of the field magnitude along a field line. For an elementary electron displacement $\overrightarrow{d l}$ 
this energy is;

$$
d w=\vec{F} \cdot \overrightarrow{d l}=q \vec{E} \cdot \overrightarrow{d l}
$$

When the free electrons follow the field lines, $\overrightarrow{d l}$ becomes an element of the field line, which is collinear to the electric field $\vec{E}$. The dot product of (1) disappears, the elementary energy is expresses as a function do the field magnitude at any point of the field line.

$$
d w=q|\vec{E}||\overrightarrow{d l}|
$$

Consequently, the energy acquired by an electron moving on a field line from point $A$ to point $B$ is equal to:

$$
W=\int_{A}^{B}|\vec{F}||\vec{d} l|=q \int_{A}^{B}|\vec{E}||\overrightarrow{d l}|=q\left(V_{B}-V_{A}\right)
$$

This energy does not depend on the variation of the magnitude of the electric field between points $A$ and $B$ of the field line, but only on the potential difference between the considered points.

The simplified approach presented above, made for a voltage corresponding to the electronic avalanche threshold, shows that the collisions with the molecules of the gas limit the free electron speed. Consequently, the inertia forces related to the curvature of the trajectory, remain low relatively to the Coulomb ones, at atmospheric pressure. The Paschen's law can be used, substituting the field line length to the distance between the plane electrodes of Paschen's experiences. This equivalence is not valid for very low pressures that remains over the Paschen's minimum. For lower PDIV, Coulomb forces are also lower, but the maximum free electron speed remains the same. Consequently, the forces due to electron inertia are no longer negligible, comparing to the Coulomb forces. The trajectories of free electrons is no longer superimposed to the field lines. Equation (3) is no longer valid. In this case, more detailed simulations must be performed for estimating the kinetic energy of free electrons in the field force.

\subsection{Application of Pashen's law}

Paschen's formulae (4) predicts the threshold potential $V_{t h}$ corresponding to the ignition of the breakdown process in a gas at a pressure $p$ for a field line length $d$ in the gas [11].

$$
V_{t h}=\frac{B(p d)}{C+\ln (p d)} \quad \text { with } \quad C=\ln \left[\frac{A}{\ln (1+1 / \gamma)}\right]
$$

The Paschen's formulae uses the old unit for the pressure (Torr); the constants A and B use these units. For

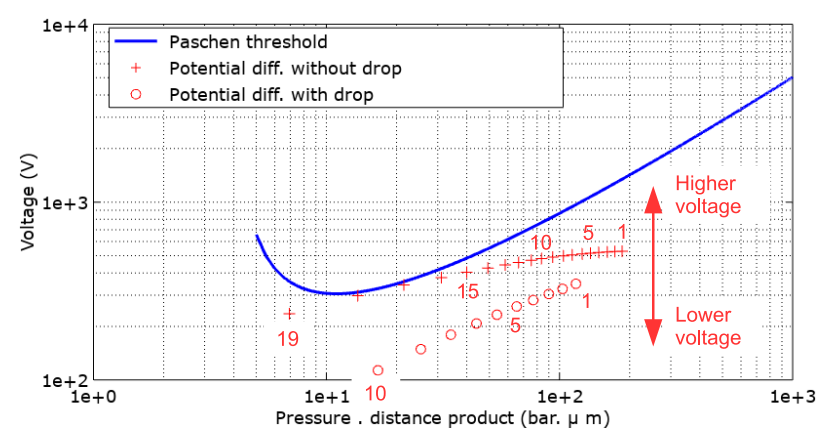

Figure 8: Voltage difference between the ends of the field lines of the critical zone in air and Paschen curve ( + initial geometry: 0 : geometry with the additional polymer drop)

dry air, at room temperature, $A=15 \mathrm{Torr}^{-1} \cdot \mathrm{cm}^{-1}, B=$ $365 \mathrm{~V}$. Torr ${ }^{-1} . \mathrm{cm}^{-1}$; the second Townsend coefficient is $\gamma=0.01$ [12]. Figure 8 plots the Paschen's curve and red points corresponding to the simulation results are added. Each + location is computed for the corresponding field line drawn in figure 5 . The simulation was performed for a voltage equal to the experimental PDIV measured on a twisted pair recovered with a silver conducting paint. Fig. 8 shows that simulations points computed for the field lines 17 and 18 are very close to the Paschen curve. For a slightly higher voltage, PDs appear between these lines, which length are respectively $13 \mu \mathrm{m}$ and $21 \mu \mathrm{m}$. The Paschen's predictions are very close to the measurements.

\section{Increase of the PDIV}

The additional conducting layer on the insulating polymer of the wire limits the critical zones to small volumes at each ends of the wire. These critical zones are well localized. A small ball of insulating varnish placed in this zones change greatly the field lines in air. Fig. 9 shows the field lines obtained with a small drop of varnish, which relative permittivity is 4 . The shortest field lines are now inside the polymer drop, which have a much higher breakdown voltage. PDs may appear in air between field lines 1 and 11 in figure 9 . The corresponding small red circles are plotted on the Pashen's graph of figure 8. A larger distance is observed between these small circles and the Paschen's curve; the voltage can be increase by about $400 \mathrm{~V}$ without any PD.

The research work it still at the very beginning of the maturation process; the technology readiness level can be estimated to TRL 2 - concept formulated.For getting the next TRL level (proof of concept), two major drawback 


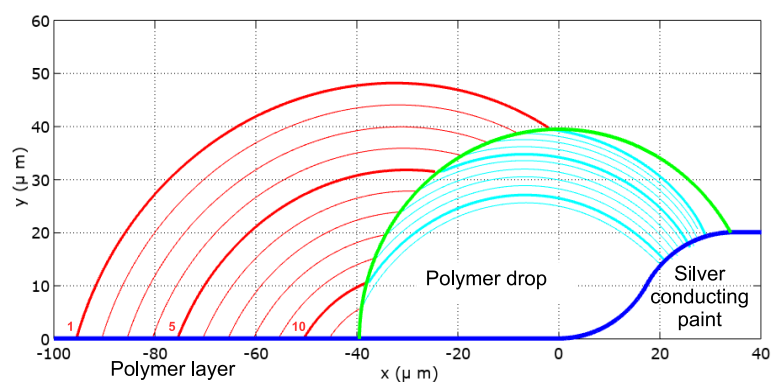

Figure 9: Field lines in the critical zone of air and in the polymer drop used to reinforce the insulation

must be overcome:

- The silver paint spread on a flexible enameled wire is brittle; such a wire cannot withstand the mechanical stress of winding machines.

- The hand made silver paint layer is very thick; its electrical resistance is low. Such an additional layer on the wire used in a machine winding would create short-circuited turns flown by large eddy currents that would create large additional Joule losses.

The application of the proposed principle in electrical motor windings require to develop an new enameled wire with a semi-conducting layer able to bring a similar electric field distribution with a high resistance per shortcircuited turn.

\section{Conclusion}

The proposed solution, consisting in adding a thin conducting layer on the external surface of an enameled wire, removes PDs in the residual air between the turns of motor coils. The additional layer moves PDs towards critical zones, near the coil connections. A careful design of these connections, with additional small balls of varnish, may increases the PDIV of the coil. However, it is not possible to built motor coils with the silver conducting paint used for preliminary experiments because its too high conductivity. For getting the maturity level TRL3 - proof of concept, experimental and theoretical developments must be performed with a thin semi-conductive external layer. A balance between the extra Joule losses and the higher PDIV must be found in close collaboration with a manufacturer of enameled wire.

\section{References}

[1] Yin W., Failure mechanism of winding insulations in inverter-fed motors, IEEE Electr. Insul. Mag. 1977, 13(6), 18-23.

[2] Fabiani D., Montanari G.C., Contin, A., Aging acceleration of insulating materials for electrical machine windings supplied bypwm in the presence and in the absence of partial discharges, Proc. Conf. ICSD, 2001, 283-286.

[3] Maussion P., Picot A., Chabert M., Malec D., Lifespan and aging modeling methods for insulation systems in electrical machines: A survey, Proc. WEMDCD, 2015, 279-288.

[4] Lahoud N., Faucher J., Malec D., Maussion P., Electrical aging of the insulation of low-voltage machines: Model definition and test with the design of experiments, IEEE Trans. Ind. Elect.2013, 60(9), 4147-4155.

[5] Iosif V., Duchesne S., Roger D., Voltage stress predetermination for long-life design of windings for electric actuators in aircrafts, Proc. Conf. CEIDP, 2015, 319-321.

[6] Duchesne S., Mihaila V., Vélu G., Roger D., Study of wire distribution in a slot of a motor fed by steep fronted pulses for lifetime extension, Proc. Conf. ISEI, 2012, 601-605.

[7] Misak S., Hamacek L., Bartłomiejczyk M., Verification ofa novel method of detecting faults in medium-voltage systems with covered conductors, Polish Ac. Sc., Metrology \& Meas. Syst., 2017, 24(2), 277-288.

[8] Stone G., Boulter E., Culbert I., Electrical insulation for rotating machines, design, evaluation, aging, testing, and repair, 2004, John Wiley \& Sons

[9] Standard EN 60172, Méthoded'essai pour la détermination de l'indice de température des fils de bobinageémaillés, 1994

[10] http://syntronics.net/coronafinder.html.

[11] Paschen F., Ueber die zumfunkenübergang in luft, wasserstoffund kohlensäurebeiverschiedenendruckenerforderliche potential differenz, Annalen der Physik, 1889, 273(5), 69-96.

[12] Townsend J.S., The theory of ionization of gases by collision, 1910, Constable \& Company

[13] Hutton J.G., Determination or corona starting voltages or nonuniform fields in air, AIEE Trans., 1947, 66(1), 1674-1680.

[14] Smalo H.S., Astrand P.O., Ingebrigtsen S., Calculation of ionization potentials and electron affinities for molecules relevant for streamer initiation and propagation, IEEE Trans. Dielect. Elec. Ins., 2010, 17(3), 733-741. 